Bio - grafía. Escritos sobre la Biología y su Enseñanza. ISSN 2027-1034

Edición Extraordinaria. p.p. 827 - 833

Memorias del IX Encuentro Nacional de Experiencias en Enseñanza de la Biología y la Educación Ambiental. IV Congreso Nacional de Investigación en Enseñanza de la Biología.

\title{
ARTRÓPODOS COMO MODELO BIOLÓGICO PARA DAR CUENTA DE LA IMPORT ANCIA DE LOS INVERTEBRADOS EN EL MEDIO AMBIENTE A TRAVÉS DE LA ENSEÑANZA DE SU ECOLOGÍA
}

\section{ARTHROPODS AS A BIOLOGICAL MODEL TO ACCOUNT FOR THE IMPORTANCE OF INVERTEBRATES IN THE ENVIRONMENT THROUGH TEACHING THEIR ECOLOGY}

\author{
Mayorga-Palacio Marisol \\ Flórez-Abreu Steeven ${ }^{1}$
}

\section{RESUMEN}

La ecología es el estudio de las relaciones de un organismo con su ambiente, en particular el de las interacciones con las plantas y animales con los que convive, esta ponencia nos presenta el uso de artrópodos como una estrategia para generar una visión inicial de la importancia de los invertebrados en el medio ambiente, dirigida a estudiantes de grado tercero pertenecientes a la Institución Educativa Distrital Colegio Alejandro Obregón a partir de la comprensión, reconocimiento de las características , e importancia ecológica de los artrópodos, a través del desarrollo de actividades planteadas y ejecutadas a lo largo de la implementación de la unidad didáctica. Se emplean elementos de recolección de información de carácter cualitativo; tales como entrevistas, observación participativa, diario de campo entre otros, y partiendo del principio de aprendizaje significativo propuesto por Ausubel que menciona la importancia de tener a consideración la concepción que tienen los niños de un determinado tema. Como resultado se obtiene una comprensión inicial en cuanto a la importancia ecológica de las funciones que desempeñan los Miriápodos, arácnidos, crustáceos e insectos en los ecosistemas, tales como la polinización, el control biológico, la descomposición de materia orgánica y como fuente de alimento por parte de los estudiantes.

PALABRAS CLAVE: ecología, artrópodos, aprendizaje significativo

\section{SUMMARY}

Ecology is the study of the relationships of an organism with its environment, in particular the study of relations with plants and animals with what it coexists, this paper does not present the use of strategies as a strategy to generate awareness of importance Of invertebrates in the environment, aimed at third grade students belonging to the Alejandro Obregon School District Educational Institution based on the understanding and recognition of the characteristics, use and ecological importance of arthropods, through the development of The activities raised and implemented A long implementation of the didactic unit. Use of data collection elements of a qualitative nature; Tales such as

\footnotetext{
${ }^{1}$ Licenciatura en Biología. Universidad Distrital Francisco José de Caldas
} 


\title{
Bio - grafía. Escritos sobre la Biología y su Enseñanza. ISSN 2027-1034
}

\section{Edición Extraordinaria. p.p. 827 - 833}

\author{
Memorias del IX Encuentro Nacional de Experiencias en Enseñanza de la Biología y la \\ Educación Ambiental. IV Congreso Nacional de Investigación en Enseñanza de la \\ Biología.
}

interviews, participatory observation, field diary among others, starting from the principle of meaningful learning proposed by Ausubel that mentions the importance of having a consideration the conception that children have of a certain subject. As a result, an understanding of the ecological importance of the functions of myriapods, arachnids, crustaceans and insects in ecosystems is obtained, such as pollination, biological control, and decomposition of organic matter and the source of food by Part of the students

KEY WORDS: ecology, arthropods, meaningful learning

\section{INTRODUCCIÓN}

A pesar del interés en las relaciones entre los seres humanos, el medio ambiente y la importancia del bienestar de tales relaciones, persisten serios problemas ambientales y de conservación (Pizarro, López, Jewell \& Inostrosa, 2014). Una razón es que casi la mitad de la población mundial vive en áreas urbanas y está cada vez más desconectada de la naturaleza (Miller, 2005). Este alejamiento podría comenzar a temprana edad, y se incrementa con el paso de los años, reconociéndose que los momentos críticos para el conocimiento e integración hacia la naturaleza y el respeto por esta son la niñez y la adolescencia (Pizarro et al.,2014). En este sentido, se ha propuesto que la educación a la conservación se desarrolla durante la niñez, y que las experiencias con el medio ambiente durante esta etapa son muy importantes en crear y mejorar las percepciones de los ambientes naturales y de los animales (Pizarro et al., 2014)

A partir de ellos, se ha observado que en los últimos años existe una fuerte tendencia en articular la enseñanza de la ecología en los diferentes niveles de educación (Primaria y Secundaria), ya que basa su razón de estudio en las relaciones de un organismo con su ambiente inorgánico y orgánico, en particular el estudio de las relaciones de tipo positivo y de tipo negativo con las plantas y animales con las que convive, esto con el fin de lograr una visión global y unitaria de la realidad (Sureda\& Colom1989). En ese aspecto se debe mencionar que la problemática que se introduce en la actualidad al considerar la ecología y la conservación de la biodiversidad es el grupo de seres vivos hacia los que se dirige la atención. Normalmente, en la mayoría de los casos, la protección de un área o la acción de preservar una especie se basa exclusivamente en datos sobre la flora o bien en estudios sobre animales vertebrados, despreciando al más importante componente de la diversidad biológica de cualquier ecosistema, los invertebrados (Arnaldos, García \& Presa 2010).

Resulta muy importante la enseñanza de artrópodos para enriquecer la perspectiva limitada que se tiene de su morfología, distribución, ecología e importancia. Los artrópodos, cuyo nombre deriva del hecho de que tienen el cuerpo segmentado son animales invertebrados que incluyen una gran variedad de especies, clases y órdenes. Prestan diversos beneficios a los ecosistemas en los que viven. Algunos por ser herbívoros, participan en el flujo de energía y nutrientes (escarabajos), otros actúan en la polinización de las plantas (mariposas y abejas), mientras que otros sirven de alimento para otras especies. Algunos, tienen la capacidad de regular poblaciones de otros 
Bio - grafía. Escritos sobre la Biología y su Enseñanza. ISSN 2027-1034

Edición Extraordinaria. p.p. 827 - 833

Memorias del IX Encuentro Nacional de Experiencias en Enseñanza de la Biología y la

Educación Ambiental. IV Congreso Nacional de Investigación en Enseñanza de la Biología.

organismos, prendado y parasitando, se constituyen en la actualidad como controladores biológicos disminuyendo el uso de productos químicos los cuales han afectado drásticamente a los espacios naturales y a los elementos que lo conforman (Shepardson, 2002). Respecto al grado de conocimiento que se tiene frente a la enseñanza de la ecología de los artrópodos, Jiménez (1998) plantea que los estudiantes presentan vacíos conceptuales sobre los animales, por ejemplo; les otorgan clasificaciones incorrectas, tienen una visión distorsionada sobre la alimentación, desconocen el hábitat, y conceden caracteres negativos. Finalmente, estas dificultades son caracterizadas y atribuidas a producciones sociales como las procedentes del ámbito familiar entre otras. A su vez Shepardson (2002) en su investigación "Bichos, mariposas y arañas: comprensión de los niños sobre los insectos". demuestra que los niños no identifican algunas características morfológicas de los insectos, por lo cual clasifican animales dentro de este grupo que no corresponden, utilizan términos (bichos) inapropiados para referirse a los insectos, proporcionan atributos dañinos a los insectos desconociendo en su totalidad los beneficios que estos proporcionan. Las ideas sobre los hábitos de alimentación de los insectos son unidireccionales, es decir que reconocen que un insecto puede ser depredador, pero no presa. El trabajo con los niños puede orientarse hacia la conciencia ambiental y conservacionista de estas especies. Se debe enfatizar en la apertura de escenarios educativos que permitan comprender las funciones de los artrópodos en los ecosistemas, como la polinización, el control biológico, la descomposición y como fuente de alimento. Resulta importante hacer uso del aprendizaje significativo como método de enseñanza de la ecología ya que los principios de este aprendizaje planteados por Ausubel (1983) proponen el diseño de herramientas que nos permitan reconocer la estructura cognitiva que poseen los estudiantes, de esta manera se parte desde un punto de referencia conceptual del estudiantado valorando sus ideas previas, esta identificación permitirá una mejor labor educativa ya que el docente construye conjuntamente con el estudiante es decir, "no parte de mentes en blanco sino de un conocimiento previo que se usa como base para la transformación y consolidación del conocimiento que se desea alcanzar" (Ausubel, 1983 p.1).

El objetivo de este trabajo es generar un elemento que aporte de manera significativa en la comprensión de la importancia de los artrópodos, incentivar el respeto hacia este grupo y generar una actitud favorable de cuidado a partir de la enseñanza de su ecología.

\section{METODOLOGÍA}

La investigación se desarrolla con base a la metodología cualitativa la cual, Elsy Bonilla (2013), afirma que esta permite que se interprete la realidad de forma descriptiva, analítica, critica, objetiva y subjetiva. El investigador cualitativo debe involucrarse con la población de estudio para tener una interpretación más objetiva y real de los acontecimientos que desea explicar, también debe procurar interactuar con la población de estudio para lograr identificar puntos críticos y problemáticas que se presenten en diferentes áreas que afectan a las poblaciones. 


\section{Bio - grafía. Escritos sobre la Biología y su Enseñanza. ISSN 2027-1034}

\section{Edición Extraordinaria. p.p. 827 - 833}

Memorias del IX Encuentro Nacional de Experiencias en Enseñanza de la Biología y la Educación Ambiental. IV Congreso Nacional de Investigación en Enseñanza de la Biología.

Esta investigación se realizó en la Institución Educativa Distrital Colegio Alejandro Obregón, Bogotá D.C; dicha institución presta servicios de Básica primaria, secundaria y media. El grupo con quien se desarrolló el trabajo corresponde a estudiantes de tercero de primaria con edades entre 8 y 10 años. Las técnicas o instrumentos para la recolección de información se basaron en la observación participante en la cual el investigador se integra a la comunidad con el objeto de hacer parte de ella y facilitar el trabajo de recolección de datos. Los datos se obtuvieron a partir de la elaboración de un diario de campo y por medio de la implementación de dispositivos mecánicos como: grabadora y cámara fotográfica.

En el marco del desarrollo de esta investigación cualitativa se realizó como primera medida la caracterización socioeconómica de los estudiantes, además de un cuestionario que nos permitió dar cuenta de los conceptos previos de los estudiantes acerca de los artrópodos. Posterior a ello se implementaron 4 sesiones de clase con diferentes actividades; en la primera sesión se dio a conocer los conceptos y elementos que caracterizan al grupo de invertebrados, como actividad se pidió a los estudiantes realizar un dibujo libre de tres invertebrados que fueran de su agrado bien sea por las características morfológicas que posean o por algún tipo de vínculo emocional que presentaran con el individuo. En la segunda y tercera sesión se enfatizó en explicar las características que presentan los artrópodos, como su morfología, sus hábitos alimenticios, sus hábitos de cortejo y sus respectivos ciclos de vida; como actividad se aplicó un cuestionario que diera cuenta del aprendizaje de los estudiantes en cuanto a los artrópodos. En la sesión final se realizaron exposiciones por parte de los estudiantes, en donde debían escoger un individuo perteneciente al grupo de artrópodos, y explicar sus características ecológicas, así como su importancia ambiental. Todas las intervenciones se iniciaron por medio de una construcción a partir de preguntas que requirieron la participación de los estudiantes entorno a lo que conocen, sus experiencias y deseos de saber, y el conocimiento de los encargados de la clase.

\section{RESULTADOS Y ANÁLISIS}

La realización del cuestionario de conceptos previos por parte de los estudiantes acerca de los artrópodos, puso en evidencia que estos reconocen que un artrópodo es un invertebrado por la "ausencia de huesos", pero respecto a las características morfológicas de estos y al reconocimiento de los diferentes grupos dentro de los mismos presentan una comprensión inicial confusa como otorgar clasificaciones incorrectas tal como lo menciona Jiménez (1998), al incluir a las arañas dentro del grupo de insectos. A partir a esta concepción se pregunta a los estudiantes ¿Cuál es la diferencia entre un arácnido y un insecto? A la cual los estudiantes respondieron haciendo uso de sus experiencias más que a su construcción académica, que las arañas se diferencian de los insectos por la acción de sacar tela, y la producción de veneno, tan solo un estudiante expreso: "se diferencian porque los insectos no tienen 8 patas como las arañas". A partir de la construcción conjunta con los estudiantes se pudo establecer que estos individuos se diferencian por el número de tagmas identificando en los insectos tres tagmas (cabezatórax y abdomen) y en arañas 2 tagmas (prosoma y opistosoma) y en el número de patas, 
Bio - grafía. Escritos sobre la Biología y su Enseñanza. ISSN 2027-1034

Edición Extraordinaria. p.p. 827 - 833

Memorias del IX Encuentro Nacional de Experiencias en Enseñanza de la Biología y la

Educación Ambiental. IV Congreso Nacional de Investigación en Enseñanza de la Biología.

siendo los arácnidos un grupo aparte de insectos. En cuanto a la morfología de insectos los estudiantes solo reconocen las patas y alas como estructuras de categorización de estos individuos; por lo que es necesario dar a conocer además de los tres tagmas notables de estos insectos estructuras como: Tipo de alas, presencia de antenas, y tipo de aparato bucal tal como se hizo en las sesiones implementadas.

En cuanto a la alimentación de los artrópodos, tal como lo evidencia Díaz (2013) la mayoría de los estudiantes presentan ideas superficiales al generalizar que estos individuos se alimentan principalmente de material vegetal, otros atribuyen el néctar entendido como miel, como alimento esencial de mariposas y abejas, ignorando la descomposición de material fecal por parte de los coleópteros o el carácter predatorio de miriápodos e incluso entre los mismos artrópodos.

Partiendo de estas concepciones se inicia una construcción conceptual con los estudiantes, orientando dicha construcción con la formulación de una pregunta guía, por ejemplo: ¿Para qué sirve la seda de las arañas? Frente a esta pregunta un estudiante responde: "ellas hacen con su seda una telaraña para atraer a las moscas, para así envolverlas y alimentarse". Con base a esta respuesta se observa el reconocimiento de otro habito alimenticio por parte de los estudiantes. Seguida de esta pregunta y para dar cuenta de la importancia de las arañas en el ambiente se realiza la siguiente pregunta ¿Cuál es la importancia de las arañas en la casa y en el ambiente? Obteniendo como respuesta que "las

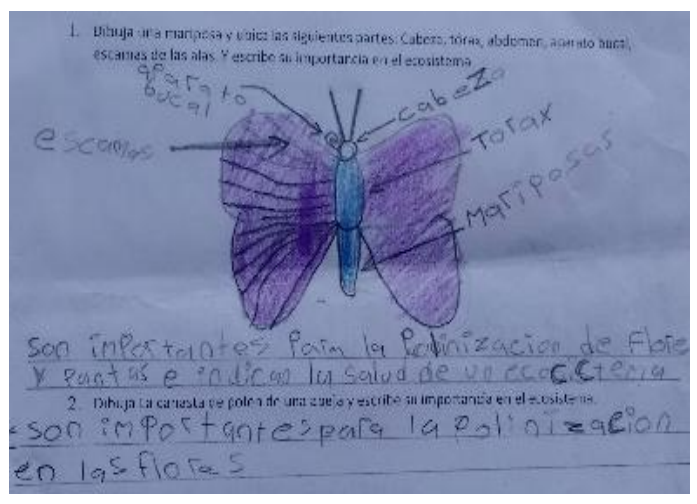
arañas se comen a los insectos que entran en la casa" evidenciando así, como el estudiante desarrolla una concepción inicial frente a la importancia de estos individuos como controladores de poblaciones biológicas.

En cuanto al hábito alimenticio de las abejas y mariposas entendiendo el néctar como miel, y el desconocimiento de la importancia del polen, se inicia la explicación morfológica del aparato bucal característico de mariposas (lepidóptera) denominado espiritrompa. Respecto a esto los estudiantes reconocieron a posterior de las clases que esta estructura se estira para alcanzar el néctar de las flores el cual es una concentración de azucares que sirve de alimento para estos individuos. Además de esto establecen la importancia de estos individuos en un proceso que podríamos considerar el inicio de la construcción del significado de polinización al reconocer que,

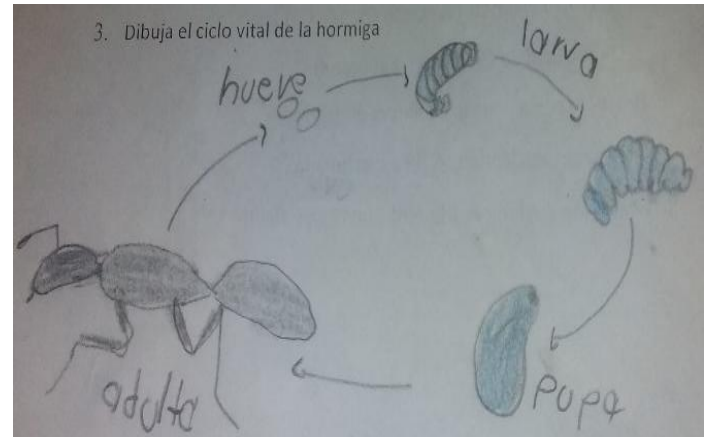

Fig. 1. Identificación de estructuras que componen el cuerpo Taller realizado posterior al proceso de enseñanza. 
Bio - grafía. Escritos sobre la Biología y su Enseñanza. ISSN 2027-1034

Edición Extraordinaria. p.p. 827 - 833

Memorias del IX Encuentro Nacional de Experiencias en Enseñanza de la Biología y la Educación Ambiental. IV Congreso Nacional de Investigación en Enseñanza de la Biología.

"en las alas membranosas, y patas de la mariposa se pega el polen de la flor ayudando en la dispersión de este y por ende a la reproducción de la flor". Fig. 1

Para dar cuenta de la importancia de la diversidad de hábitos alimenticios que poseen los artrópodos a los estudiantes se pregunta ¿De qué se alimentan los coleópteros?; un estudiante responde "Son cochinos porque se alimentan de popo", concediendo un carácter negativo tal como lo menciona Jiménez (1998). Frente a esto se realiza la explicación partiendo de una reflexión al decirles "se imaginan ustedes un mundo donde todo ese popo que producen los animales y nosotros se almacenara", dando a entender que estos cumplen una función de "limpieza" al alimentarse de algo que ningún otro ser vivo consume descomponiendo material fecal. Bajo este contexto también se menciona la importancia de los Grillos en cuanto a la descomposición de residuos vegetales como la hojarasca, los miriápodos como predadores y los crustáceos como filtradores.

Al hablar de ciclos de vida de insectos, la primera concepción que tienen los estudiantes frente al tema es el ciclo de vida de la mariposa al decir "por ejemplo la mariposa: sale el huevo, sale una oruga, hace un capucho y sale una mariposa" Esta concepción es aceptable, sin embargo, no reconocen que este ciclo de vida también lo comparten las hormigas, abejas, coleópteros, ni las transformacion parte de su ciclo de vida Fig. 3. Por lo que es necesá de la etapa larval al estadio adulto, y que existe otro ti|

Fig. 3 ciclo de vida de una hormigaconcepción después de las intervenciones.

\section{CONCLUSIÓN}

Se estableció que el uso de artrópodos constituye un adecuado modelo de enseñanza y aprendizaje frente a la importancia que poseen los invertebrados en el ambiente ya sea por el alto grado de familiaridad que se presenta con los grupos desde muy tempranas edades, la diversidad de estos y la facilidad que se posee para acceder a este tipo de organismos. Así mismo se desarrolló una visión inicial por parte de los estudiantes frente a la importancia de las funciones ecológicas como la polinización, el control biológico, la descomposición de materia orgánica y fuente de alimento que desempeñan los arácnidos, miriápodos, crustáceos e insectos para el ambiente.

\section{BIBLIOGRAFIA}

- Arnaldos, I.; García, D.; Presa, J. (2010) Artrópodos en la consenvación Universidad de Murcia. 8-16

- Ausubel, D. (1983) Teoría del aprendizaje significativo. Psicología Educativa. Editorial Trillas. México. 
Bio - grafía. Escritos sobre la Biología y su Enseñanza. ISSN 2027-1034

Edición Extraordinaria. p.p. 827 - 833

Memorias del IX Encuentro Nacional de Experiencias en Enseñanza de la Biología y la Educación Ambiental. IV Congreso Nacional de Investigación en Enseñanza de la Biología.

- Bonilla, E.; Rodríguez, P. (2013) La Investigación en Ciencias Sociales. Más allá del dilema de los métodos. Tercera edición. E-Book Bogotá- Colombia. Grupo Editorial Norma.30-50

- Díaz, Y. 2013. Diseño de una unidad didáctica para el estudio de los insectos. Tesis Universidad del Valle Instituto de Educación y Pedagogía Santiago de Cali, Colombia

- Jiménez, M. 1998. Concepciones sobre algunas especies animales: ejemplificaciones del razonamiento por categorías. Dificultades de aprendizaje asociadas. Revista Enseñanza de las Ciencias, 16(1): 174-157.

- Miller, J. 2005. Biodiversity conservation and the extinction of experience. Trends in Ecology and Evolution, 20: 430-434.

- Pizarro, J.; López, F.; Jewell, S.; Inostroza, C. 2014. Preferencias de niños y niñas en relación con los artrópodos epigeos (Metazoa: Arthropoda) del desierto florido de Chile IDESIA Chile Volumen 32, № 3.

- Sheparsond, D. 2002. Bugs, butterflies, and spiders: children`s understandings about insects. International Journal of science Education, 24

(6): 627-643. 